\title{
AIP
}

\section{Variational ground state for the periodic Anderson model}

Samuel P. Bowen and Jay D. Mancini

Citation: Journal of Applied Physics 63, 3402 (1988); doi: 10.1063/1.340774

View online: http://dx.doi.org/10.1063/1.340774

View Table of Contents: http://scitation.aip.org/content/aip/journal/jap/63/8?ver=pdfcov

Published by the AIP Publishing

\section{Articles you may be interested in}

The periodic Anderson model in the generating functional approach

AIP Conf. Proc. 789, 247 (2005); 10.1063/1.2080351

Strongly correlated electron ground-state energy approximations for Anderson-like models

J. Appl. Phys. 61, 3168 (1987); 10.1063/1.337829

Excitation of molecular rotation by periodic microwave pulses. A testing ground for Anderson localization

J. Chem. Phys. 84, 2604 (1986); 10.1063/1.450330

Density of states for the asymmetric Anderson model

J. Appl. Phys. 53, 2131 (1982); 10.1063/1.330756

Variational Definition of Anderson's Ligand Field States

AIP Conf. Proc. 18, 656 (1974); 10.1063/1.3141792

\section{MIT LINCOLN}

LABORATORY CAREERS

Discover the satisfaction of innovation and service to the nation
- Space Control

- Air \& Missile Defense

- Communications Systems \& Cyber Security

- Intelligence, Surveillance and Reconnaissance Systems

- Advanced Electronics

- Tactical Systems

- Homeland Protection

- Air Traffic Control

\section{LINCOLN LABORATORY}

MassachusetTs Institute OF TeChNOLOGY

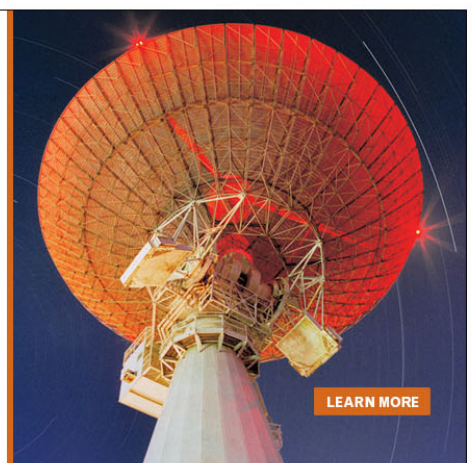




\title{
Variational ground state for the periodic Anderson model
}

\author{
Samuel P. Bowen \\ Department of Physics, Virginia Tech, Blacksburg, Virginia 24051 \\ Jay D. Mancini \\ Department of Physics, Fordham University, Bronx, New York 10458
}

\begin{abstract}
A variational calculation of the ground state of the Anderson lattice model is discussed. The calculation creates a finite Hamiltonian matrix for many-electron states, which are appropriate for the thermodynamic limit. A simple $14 \times 14$ truncation is discussed for finite $U$ and a smaller $3 \times 3$ is used for the large $U$ limit. Both approximations indicate that the spin configurations of the localized $f$ orbitals are antiferromagnetically correlated for nearest neighbors in the ground state. This antiferromagnetic correlation is mediated by an RKKY interaction and thus offers a variety of spin orderings as a function of lattice and density.
\end{abstract}

\section{INTRODUCTION}

The study of the ground state of the Anderson latice model (ALM) has recently generated several papers. ${ }^{1}$ These studies have used a variety of methods, of which the most successful to date have been variational. The most frequently used technique in the last year has been the Gutzwiller method. ${ }^{2}$ In this approach two particle states on the localized single-site $f$ states are projected out of the variational wave function. The minimization of the energy functional is carried out in the usual way. An essential assumption in most applications of the Gutzwiller wave function is the evaluation of expectation values only at single sites on the lattice. In fact, many authors have correctly characterized the Gutzwiller method as a single-site mean field theory ${ }^{3}$ in the sense that each f-orbital site is assumed to be the same as the others. Recently, Vulovic' and Abrahams demonstrated that the Hartree Fock ferromagnetic ground state was lower in energy than the Gutzwiller paramagnetic state in the Kondo regime for a certain range of model parameters. This discovery points out a weakness in the Gutzwiller approach in that no information about the spin correlations between localized $f$ orbitals at different sites is available.

This pape: presents an altemative approach to the variational problem for the ALM, which does give information about intersite correlations. The method we propose here starts from the same initial state as the Gutzwiller method, but inciudes the doubly occupied states. We obtain information about the $f$-orbital lattice spin configurations through their contributions to the ground-state energy. In particular, we find for a wide range of densities that the ground state should be dominated by a nearest-neighbor antiferromagnetic correlation. A wide variety of orderings is possible.

This method has its origin in the Mancini and Mattis ${ }^{5}$ application of the Lanczos recursion scheme ${ }^{6}$ to many-electron states. The restriction of this original Lanczos method to tridiagonal total energy matrices was relaxed ${ }^{7}$ with considerable success and improved convergence when applied to the Anderson impurity model. The truncated matrix approximations for the type of basis states to be discussed below gave much lower ground-state energies than the corresponding Mancini-Mattis tridiagonalizations with less effort. It was found in comparison with exact Bethe Anzatz gromd state of the Anderson impurity model that the simplest approximations of this approach recovered the major properties quite well. This paper represents an extension of these ideas to the ALM.

The approach starts with a suitable many-electron state and then applies the Hamilonian $M$ to it. Successive applications of $H$ creates an energy matrix between orthogonal many-electron states. The initial stages generated in this sequence have been found to contain a surprising amount of information about the ground-state correlations. Finite truncations of this matrix have provided reasonably good approximations to the ground-state properties of the Anderson impurity model with relatively little iabor. In the simplest demonstration of this property for the ALM we will show that a $3 \times 3$ matrix truncation (in the large $U$ limit) displays an antiferromagnetic correlation which lowers the ground state over ferromagnetic and random spin configurations.

A possible strength of this method is its ability to exhibit ground-state information with relatively small amounts of effort. The ground-state energies are given in terms of averages of single-particle energies and occupation probabilities over the initial states. ${ }^{7}$ As will be seen below, this method seems to give information that has not been found in other methods. One drawback of this technique is, however, that small excitation energies from near the Fermi energy are not well approximated in the initial truncations and will probably require very large basis sets. The vectors that are included in the truncations are all valid in the thermodynamic limit.

\section{METHOO}

The initial vector is the same as that used by the Gutzwiller approach, and consists of a half-filled conduction band and the lattice of singly occupied forbitals with a given spin configuration:

$$
\left|\Psi_{0}\right\rangle=A_{F}^{+}\left|s_{1}, s_{2}, \ldots\right\rangle,
$$

where the spin configuration for the lattice is specified by the set of spins on each site $\left(s_{1}, s_{2}, \ldots\right)$ and $A_{F}^{+}$creates the Fermi sea.

The ALM Hamiltonian is 


$$
\begin{aligned}
& H=\sum_{k^{\prime} s} c_{k s}^{+} c_{k s}+\sum_{i} E_{s_{1}} f_{i s_{1}}^{+} f_{1 s_{1}}+U \sum_{i} f_{i}^{+} f_{1} f_{1_{l}}^{+} f_{i_{i}} \\
& +V / \sqrt{N} \sum_{k^{\prime} s} \sum_{i}\left(e^{i k k_{1}} f_{i s_{1}}^{+} c_{k s_{1}}+H_{0} C_{0}\right),
\end{aligned}
$$

where $c_{k s}^{+}\left(c_{k s}\right)$ and $f_{1 . s}^{+}\left(f_{t s}\right)$ are the creation (annihilation) operators for the conduction band electrons and localized $f$ electrons, respectively. The conduction band energies are given by $\epsilon_{k s}$ and the f-orbital energies are given by $E_{s}$. The on-site coulomb repulsion for the localized $f$ electrons is $U$ and the hybridization matrix element is $V$ (assumed to be independent of wave vector $k$ ).

The variational calculation is begun by applying the AlM Hamiltonian to $\left\{\Psi_{0}\right\rangle$. This gives two new orthogonal many-electron states $\left\langle\Psi_{1}\right\rangle$ and $\left|\Psi_{2}\right\rangle$.

The first row of the Hamiltonian matrix in this basis is $H\left|\hat{\Psi}_{0}\right\rangle=E_{0}\left|\hat{\Psi}_{0}\right\rangle+\sqrt{N} \sqrt{x} \cdot\left|\hat{\psi}_{1}\right\rangle+\sqrt{N} \sqrt{\left(1-x_{+}\right)} \mid \hat{\Psi}_{2}$, where

$$
\begin{aligned}
\left|\hat{\varphi}_{1}\right\rangle= & 1 / N \mathbb{1} / \sqrt{\left(1-x_{+}\right)} \sum_{k^{\prime} !} e^{-i K \cdot R_{2}} \\
& \times c_{k^{\prime} s_{1}}^{+} A_{f}^{+}\left\{s_{1}, s_{2}, \cdots(\uparrow \downarrow), \cdots\right\rangle
\end{aligned}
$$

and

$$
\begin{aligned}
\left|\hat{\Psi}_{2}\right\rangle= & 1 / N 1 / \sqrt{\left(1-x_{+}\right)} \sum_{k^{\prime} l} e^{-k_{k} \cdot \mathbb{R}_{1}} \\
& \times c_{k^{\prime} s_{1}}^{+} A+\left|s_{1}, s_{2}, \cdots(0)_{1} \cdots\right\rangle,
\end{aligned}
$$

where $x_{ \pm}$is defined below in Eq. (6c). The first vector $\left|\hat{A}_{1}\right\rangle$ creates a doubly occupied $f$ state on the iattice and a conduction electron hole. The $k$ sum is over the first Brillouin zone and the $l$ sum is over the entire set of lattice sites. The second vector $\left\langle\widehat{\Psi}_{2}\right\rangle$ creates an empty $f$ orbital and a conduction electron above the Fermi energy. These two states are orthogonal and their norms are given by

$$
\begin{aligned}
& \left\langle\Psi_{1} \mid \Psi_{1}\right\rangle=N x_{-}, \\
& \left\langle\Psi_{2} \mid \Psi_{2}\right\rangle=N\left(1-x_{+}\right),
\end{aligned}
$$

where

$$
x_{ \pm}=1 / N^{2} \sum_{k^{\prime} !} n_{k^{\prime}\left( \pm s_{1}\right)}
$$

and where $n_{k s}=\left\langle\Psi_{0}\left|c_{k s}^{+} c_{k s}\right| \Psi_{0}\right\rangle$ and the sum represents the fractional occupation of the conduction band for particular spin states. Each of these vectors contains all possible conduction and felectron single particle hole excitations appropriate for consideration in the thermodynamic limit.

The operation of $H$ on each of these two vectors generates 11 new vectors. These vectors correspond to all possible two-electron and two-hole states and their distribution between the conduction band and the f-electron lattice. We may classify these 11 vectors into three distinct classes: (A) vectors that are relevant in the thermodynamic limit $(N \rightarrow \infty, \Omega \rightarrow \infty, N / \Omega$ finite), that is, those that have norms of order $N ;(B)$ vectors that are marginally relevant in the thermodynamic limit, that is, which have norms that are of order unity; (C) vectors that are not at all important in the thermodynamic limit, that is, those whose norms are of order $1 / N$. For this paper we will only include vectors of class
A in our ground-state estimates. The inclusion of all the vectors in class $B$ is required if one wants to approximate well the continum excited states of the system. Including finitely many of these class $B$ vectors in the matrix will lower the ground state a very small amount and will mostly fill in the spectrum of the excited states between the class A eigenvalues. The ciass $A$ vectors have as diagonal matrix elements of the Hamiltonian matrix average band energies and they alone should give a good approximation to the ground state.

The number of distinct vectors in class $A$ is only four. These can be further classified by their excitation structure on the $f$-orbital lattice and thus by their dependence on $U$. The $f$-electron spin lattice configuration can be represented by a set which displays the sequence of spins on exch site. A particular spin in a configuration at a site will be represented by an $s_{1}$, while an empty $f$ orbital will be represented by $(0)_{1}$, and a doubly occupied site will be denoted by $(x)_{1}$. The four types of vectors ate

$$
\begin{aligned}
& \left|s_{1}, \ldots,(0)_{1}, \ldots,(0)_{1}, \ldots, s_{n}\right\rangle, \\
& \left|s_{1}, \ldots,(x)_{1}, \ldots,(0)_{1}, \ldots, s_{n}\right\rangle, \\
& \left|s_{1}, \ldots,(0)_{1}, \ldots,(x)_{1}, \ldots, s_{n}\right\rangle, \\
& \left|s_{1}, \ldots,(x)_{1}, \ldots,(x)_{1}, \ldots, s_{n}\right\rangle .
\end{aligned}
$$

The three vectors $(7 b)-(7 d)$ all have diagonal Hamiltonian matrix elements with a $U$ term. For large $U$ the effect of these vectors on the lowest energy of the matrix will be very small. Because of size limitations on this rote we will not explicitiy consider these three vectors encept to examine some of their matrix elements below.

To examine the ground-state energy in the large $U$ limit we need only consider a matrix that has three distinct vectors: $\left|\Psi_{0}\right\rangle$, the vector in Eqs. (4) and (7a). The diagonal matrix elements are $E_{0}, E_{0}+\delta \epsilon, E_{0}+2 \delta \epsilon$, respectively, where $E_{0}$ is the energy of the conduction band and the singly occupied lattice of $f$ orbitals. The energy $\delta \epsilon$ is the average particle-hole excitation for conduction electron $f$-orbital hole excitations and is defined by

$$
\delta \epsilon=1 / N \sum_{k !}\left(E_{k s_{1}}-E_{L_{i}}\right) m_{k s:}
$$

The off-diagonal Hamiltonian matrix element between $\left|\Psi_{0}\right\rangle$ and $\left\{\Psi_{1}\right\}$ is $V \sqrt{N\left(1-x_{+}\right)}$, where $x_{\text {, }}$ is the fractional occupation number of the conduction band whin spin that is the same as the $f$-orbital configuration of $\left|\Psi_{0}\right\rangle$. The major effect to be reported in this paper is a consequence of the matrix element between the vectors in Eqs. (4) and (7a). This matrix element is

$$
\begin{aligned}
\Gamma= & {\left[\frac { N } { ( 1 - x _ { + } ) ^ { 2 } } \left(\left(1-x_{+}\right)^{2}\right.\right.} \\
& \left.\left.-\frac{1}{N} \sum_{\mathbb{R}_{11} \neq 0} \delta s_{1}, s_{1},\left|J_{s}\left(\mathbb{R}_{11}\right)\right|^{2}\right)\right]^{1 / 2},
\end{aligned}
$$

where $J_{s}(\mathbf{R})$ is the RKY function

$$
J_{s}(R)=\frac{1}{N} \sum_{k} e^{i k \cdot R_{11}^{\prime \prime} n_{k s}} .
$$

It is important to notice in Eq. (9) that the lartice sum of the squares of the RKKY functions contain only terms in which 
the two spins are parallel. This means that the second term in the matris element will be largest when all of the spins are aligned in a ferromagnetic arrangement. Such an arrangement will make the value of this off-diagonal matrix element its smallest. Any other arrangement of spins will give a larger matrix element and thus a lower ground-state energy estimate. Because the RKKY functions are largest for small separations and oscillate with increasing $R$ the effect of the nearest neighbors will be largest in the lattice sum. Thus $f$ orbital lattice spin configurations which have strong nearestneighbor antiferromagnetic correlations will result in the lowest ground-state estimates.

An approximate expression for the ground state of the $3 \times 3$ matrix mentioned above is

$$
E_{g}=E_{0}-\frac{N\left(V^{2} / \delta \epsilon\right)}{\sqrt{(1-x+)}}\left[1+(\Gamma / \delta \epsilon)^{2}+\cdots\right]
$$

where we have expanded in a series expansion to lowest order in the matrix element ( 9 ). As discussed above, in a ferromagnetic lattice $\Gamma$ is its smallest. In an antiferromagnetic configuration $\Gamma$ will be its largest and the ground state will be at its lowest.

This conclusion about the f-orbital spin configurations is not altered by the inclusion of the vectors ( $7 b)-(7 d)$. The remaining matrix elements in the extended matrix, which are necessary for a finite $U$ discussion are of the form

$$
\sqrt{N / x_{-}}\left(x_{-}\left(1-x_{+}\right)+\frac{1}{N} \sum_{R_{11 ; \neq 0} \delta} \delta_{-s_{1}, s_{1}}\left|J\left(R_{11^{\prime}}\right)\right|^{2}\right)^{1 / 2} \text {. }
$$

Note that here the RKKY factor lattice sums only contain contributions from pairs of $f$ orbitals with antiparallel spins and the sums in this case increase the size of the matrix elements. In this case larger matrix elements also will be given by antiferromagnetic correlations, especially between neighboring sites. These larger matrix elements will further contribute to a lower ground-state energy.

The RKKY functions have a scale factor determined by the Fermi wave vector of the conduction band. This gives an indirect dependence on the lattice spacing. In real lattices, these functions will reflect the symmetry of the Fermi surface and have different values for different crystal directions. It is possible that a great variety of spin configurations could be consistent with the lowest ground state depending on the location of the zeros of the RKKY functions and the spacing of the lattice sites.

\section{SUMMARY}

In this work we have applied a discrete matrix method to the variational calculation of the ground-state energy of the ALM. While this method is not extremely sensitive to excitations close to the Fermi energy it nevertheless gives information about ground-state spin correlations which are induced by the interplay of the hybridization and the Coulomb repulsion. The results of this work are valid for finite $U$. One of the more surprising aspects of this study is that the size of $U$ does not aiter the qualitative conclusion that antiferromagnet correlations among the localized $f$ spins dominates the ground state of the ALM. Matrix elements which are operable for finite $U$ also favor nearest-neighbor antiferromagnetic correlations. This variational method is not dependent on the dimensionality of the system and a comparison of the ground states in different dimensions will be discussed elsewhere. Another aspect of this approximation which needs to be explored further is the variety of spin configurations and crystal structures that arise out of the interplay of the range and symmetry of the RKKY functions.

\section{ACKNOWLEDGMENT}

One of the authors (IDM) would like to thank Dr. Judith Ginsberg of the Research Council of Fordham University for partial support of this work.

'B. H. Brandow, Phys. Rev. B 33, 215 (1986); R. Blankenbecler, J. R. Fulco, W. Gill, and D. J. Scalapino, Phys. Rev. Lett. 58, 411 (1987); H. Shiba, J. Phys. Soc. Jpu. 55, 2765 (1986).

2P. Fazekas, J. Magn. Magn. Mater. 63\&64, 545 (1987); T. M. Rice and K. Ueda, Phys. Rev. B 34, 6420 (1987); A. Oguchi, Prog. Theor. Phys. 77, $278(1987)$.

${ }^{3}$ C. M. Varma, W. Weber, and L. J. Randall, Phys. Rev, B 33, 1015 (1986). ${ }^{4}$ V. Z. Vulvovic' and E. Abrahams, Phys. Rev, B 36, 2614 (1987).

5. D. Mancini and D. C. Mattis, Phys. Rev. B 31,7440 (1985); 129,6988 $(1984) ; \mathrm{B} 28,6061(1983)$.

6R. Haydock, V. Heine, and M. J. Kelly, J. Phys. C 5, 2591 (1975).

7. D. Mancini, C. D. Potter, and S. P. Bowen, J. Appl. Phys. 61, 3186 (1987); S. P. Bowen, J. D. Mancini (unpublished). 